\title{
Clinical subtypes of core premenstrual disorders: A Delphi survey
}

Khaled M K Ismail *,1,2, Tracy Nevatte ${ }^{*, 3}$ Shaughn O’Brien ${ }^{3}$, Elena Paschetta ${ }^{2}$, Torbjorn Bäckström ${ }^{4}$, Lorraine Dennerstein ${ }^{5}$, Elias Eriksson ${ }^{6}$, Ellen W. Freeman ${ }^{7}$, Nick Panay ${ }^{8}$, Teri Pearlstein ${ }^{9}$, Andrea Rapkin ${ }^{10}$, Meir Steiner ${ }^{11}$, John Studd $^{8}$ and Inger Sundström-Poromma ${ }^{12}$.

* Joint first authors

Corresponding author: $\quad$ Professor Khaled Ismail $M S c, M D, P h D, F R C O G$ Birmingham Women's Hospital NHS Foundation Trust Edgbaston Birmingham, B15 2TG

Affiliation:

UK

1. School of Clinical \& Experimental Medicine, College of Medical \& Dental Sciences, University of Birmingham, UK

2. Birmingham Women's Hospital NHS Foundation Trust, Birmingham, UK

3. Institute for Science and Technology in Medicine, Guy-Hilton Research Centre. Keele University, UK

4. Umeå Neurosteroid Research Center, Department of Clinical Sciences, Umeå University, Sweden

5. Department of Psychiatry, University of Melbourne and National Ageing Research Institute, Australia

6. Department of Neuroscience and Physiology, Sahlgrenska Academy, University of Gothenburg, Sweden

7. Departments of Obstetrics/ Gynaecology and Psychiatry, University of Pennsylvania, USA 
8. Department of Obstetrics and Gynaecology, Chelsea and Westminster Hospital, UK

9. Warren Alpert Medical School of Brown University, USA

10. David Geffen School of Medicine at UCLA, USA

11. Departments of Psychiatry \& Behavioural Neurosciences and Obstetrics \& Gynecology, McMaster University, Canada

12. Department of Women's and Children's Health, Uppsala University, Sweden

Keywords: Premenstrual disorder

Core premenstrual disorder

Premenstrual syndrome

Premenstrual dysphoric disorder

Delphi method 
Table 1: Percentage of group voting for each score category and the Mean and median scores for the proposed clinical subtypes in Delphi rounds

$(n=12)$.

\begin{tabular}{|c|c|c|c|c|c|c|c|c|c|c|c|c|c|c|c|c|c|c|}
\hline \multirow{3}{*}{ Proposed subtypes } & \multicolumn{9}{|c|}{ First iteration } & \multicolumn{9}{|c|}{ Second iteration } \\
\hline & \multicolumn{6}{|c|}{ Percentage (\%) of group voting for each score category } & \multirow{2}{*}{$\overrightarrow{\stackrel{\vec{\theta}}{\ddot{\Xi}}}$} & \multirow{2}{*}{$\frac{3}{3}$} & \multirow{2}{*}{ 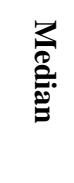 } & \multicolumn{6}{|c|}{ Percentage (\%) of group voting for each score category } & \multirow{2}{*}{$\overrightarrow{0}$} & \multirow{2}{*}{ 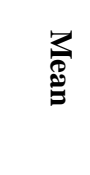 } & \multirow{2}{*}{ 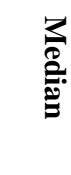 } \\
\hline & 1 & 2 & 3 & 4 & 5 & 6 & & & & 1 & 2 & 3 & 4 & 5 & 6 & & & \\
\hline $\begin{array}{c}\text { A- Premenstrual } \\
\text { Dysphoric Disorder }\end{array}$ & 0 & 0 & 0 & 8.3 & 8.3 & 83.3 & 69 & 5.75 & 6 & 0 & 0 & 0 & 0 & 8.3 & 91.7 & 71 & 5.92 & 6 \\
\hline $\begin{array}{l}\text { B- Predominantly } \\
\text { psychological }\end{array}$ & 0 & 0 & 0 & 8.3 & 41.7 & 50 & 65 & 5.42 & 5.5 & 0 & 0 & 0 & 8.3 & 25 & 66.7 & 67 & 5.58 & 6 \\
\hline $\begin{array}{l}\text { C- Predominantly } \\
\text { physical }\end{array}$ & 0 & 0 & 0 & 8.3 & 33.3 & 58.3 & 66 & 5.50 & 6 & 0 & 0 & 0 & 8.3 & 25 & 66.7 & 67 & 5.58 & 6 \\
\hline $\begin{array}{c}\text { D- Predominant breast } \\
\text { tenderness }\end{array}$ & 33.3 & 8.3 & 16.7 & 8.3 & 25 & 8.3 & 37 & 3.08 & 3 & 25 & 25 & 16.7 & 0 & 25 & 8.3 & 36 & 3.00 & 2.5 \\
\hline $\begin{array}{l}\text { E-Predominant } \\
\text { bloatedness }\end{array}$ & 41.7 & 8.3 & 8.3 & 33.3 & 8.3 & 0 & 31 & 2.58 & 2.5 & 41.7 & 16.7 & 33.3 & 8.3 & 0 & 0 & 25 & 2.08 & 2 \\
\hline $\begin{array}{c}\text { F- Predominant weight } \\
\text { gain }\end{array}$ & 66.7 & 8.3 & 25 & 0 & 0 & 0 & 19 & 1.58 & 1 & 91.7 & 0 & 8.3 & 0 & 0 & 0 & 14 & 1.17 & 1 \\
\hline $\begin{array}{c}\text { G- Predominant change } \\
\text { of appetite }\end{array}$ & 75 & 8.3 & 16.7 & 0 & 0 & 0 & 17 & 1.42 & 1 & 83.3 & 8.3 & 8.3 & 0 & 0 & 0 & 15 & 1.25 & 1 \\
\hline $\begin{array}{l}\text { H- Predominant } \\
\text { swelling }\end{array}$ & 50 & 16.7 & 33.3 & 0 & 0 & 0 & 22 & 1.83 & 1.5 & 66.7 & 33.3 & 0 & 0 & 0 & 0 & 16 & 1.33 & 1 \\
\hline $\begin{array}{l}\text { I- Predominant } \\
\text { irritability }\end{array}$ & 25 & 8.3 & 33.3 & 33.3 & 0 & 0 & 36 & 3.00 & 3 & 33.3 & 8.3 & 33.3 & 16.7 & 8.3 & 0 & 32 & 2.67 & 3 \\
\hline
\end{tabular}




\begin{tabular}{|c|c|c|c|c|c|c|c|c|c|c|c|c|c|c|c|c|c|c|}
\hline $\begin{array}{l}\text { J- History of mood } \\
\text { disorder }\end{array}$ & 50 & 8.3 & 0 & 8.3 & 16.7 & 16.7 & 34 & 2.83 & 1.5 & 50 & 16.7 & 16.7 & 0 & 0 & 16.7 & 28 & 2.33 & 1.5 \\
\hline K- Predominant fatigue & 91.7 & 8.3 & 0 & 0 & 0 & 0 & 13 & 1.08 & 1 & 100 & 0 & 0 & 0 & 0 & 0 & 12 & 1.00 & 1 \\
\hline $\begin{array}{c}\text { L- Predominant } \\
\text { depression }\end{array}$ & 50 & 8.3 & 16.7 & 16.7 & 0 & 8.3 & 28 & 2.33 & 1.5 & 50 & 25 & 8.3 & 8.3 & 0 & 8.3 & 25 & 2.08 & 1.5 \\
\hline
\end{tabular}





\section{University Library}

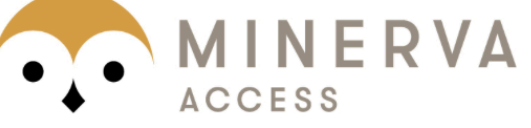

A gateway to Melbourne's research publications

Minerva Access is the Institutional Repository of The University of Melbourne

\section{Author/s:}

Ismail, KMK;Nevatte, T;O'Brien, S;Paschetta, E;Backstrom, T;Dennerstein, L;Eriksson,

E;Freeman, EW;Panay, N;Pearlstein, T;Rapkin, A;Steiner, M;Studd, J;Sundstrom-Poromaa, I

Title:

Clinical subtypes of core premenstrual disorders: a Delphi survey

Date:

2013-06-01

\section{Citation:}

Ismail, K. M. K., Nevatte, T., O'Brien, S., Paschetta, E., Backstrom, T., Dennerstein, L., Eriksson, E., Freeman, E. W., Panay, N., Pearlstein, T., Rapkin, A., Steiner, M., Studd, J. \& Sundstrom-Poromaa, I. (2013). Clinical subtypes of core premenstrual disorders: a Delphi survey. ARCHIVES OF WOMENS MENTAL HEALTH, 16 (3), pp.197-201. https:// doi.org/10.1007/s00737-012-0326-7.

Persistent Link:

http://hdl.handle.net/11343/283208 